\title{
Role of Vulcanizing Additives on the Segmental Dynamics of
}

\section{Natural Rubber}

\author{
Marianella Hernández ${ }^{1 *}$, Tiberio A. Ezquerra ${ }^{2}$, Raquel Verdejo ${ }^{1}$, Miguel A. López- \\ Manchado $^{1^{*}}$ \\ ${ }^{1}$ Instituto de Ciencia y Tecnología de Polímeros, ICTP-CSIC, Juan de la Cierva 3, Madrid 28006, Spain. \\ ${ }^{2}$ Instituto de Estructura de la Materia, IEM-CSIC, Serrano 119, Madrid 28006, Spain. \\ *corresponding authors: marherna@ictp.csic.es or 1manchado@ictp.csic.es
}

\begin{abstract}
The influence of the addition of vulcanizing additives on the molecular dynamics of unvulcanized Natural Rubber (NR) has been studied by broadband dielectric spectroscopy. Results reveal a slowdown of the segmental dynamics of NR, restricting the motion of the chains tightly bounded to the additive surfaces. In general, when zinc oxide is present a second dynamic process is detected, ascribed to strong interfacial interactions between the zinc ionic clusters and the NR polymer segments. The study of such interactions prior to vulcanization is a very useful strategy to control the vulcanization process, maximizing its benefits and, hence, improving the final products. Here, we demonstrate that broadband dielectric spectroscopy is a good experimental alternative in order to obtain a deeper insight into the vulcanization mechanisms. Further, our results support one previously proposed sulfur vulcanization mechanism, in which molecules of accelerators, sulfur and fatty acids are adsorbed on the zinc oxide surface.
\end{abstract}




\section{INTRODUCTION}

Natural Rubber (NR) holds a unique place in rubber technology due to its outstanding tack and strength in the unvulcanized state; and high tensile strength and crack growth resistance once vulcanized. ${ }^{1}$ For most applications, it is necessary to convert the rubbery linear polymer into a three-dimensional network in order to assure a complete recovery after deformation. Such a network is the result of cross-linking, covalent bonding among some chain segments, by means of temperature and pressure in which an essentially fluid material is transformed into a fully elastic one. This process is commonly known as vulcanization. ${ }^{1}$ The sulfur vulcanization is the traditional method for polydiene rubbers, including NR; however, although sulfur vulcanization is a very old large-scale industrial process, and has been considerably studied over the last several decades, a complete fundamental understanding of this complex chemical process remains a significant scientific challenge. A rigorous research effort continues today to unravel the mechanisms of the individual steps in the sulfur vulcanization process. ${ }^{2,3}$ The difficulties encountered in this research field must be accredited to a combination of diversity and complexity. This process usually involves several chemicals such as a vulcanizing agent (sulfur (S)), an accelerator, and an activator complex formed by a fatty acid (stearic acid (SA)) and zinc oxide $(\mathrm{ZnO})$. The situation is further complicated by the interaction of the accelerators and activators, each component influencing the reactivity of the other, and the way these interactions affect the vulcanization mechanism.

Plenty of literature ${ }^{1,4-9}$ reveals that the addition of these chemicals in small quantities with respect to raw rubber affects the processability and the final properties of the vulcanized compounds. In general, the use of accelerators in combination with zinc activators has a 
pronounced effect on the speed of vulcanization and on the distribution of crosslinks formed. Besides these, the durability of rubber articles as well as the physical properties, in particular the tensile strength, are very much improved by organic accelerators.

Nonetheless, the authors are struck by the fact that despite the enormous practical knowledge developed so far, a detailed description on how these constituents affect the molecular dynamics and related properties of unvulcanized NR is unknown and has not been reported until now. Moreover, most vulcanization systems have been developed by skillful and elaborated techniques based on the trial-and-error method. ${ }^{10}$ So, a detailed discussion on the relationship between molecular dynamics and the effect of each of the additives in the vulcanization system, prior to vulcanization, will be of great interest not only for technological developments, but also for academic interests. In this manuscript, we

present experimental evidence, using broadband dielectric spectroscopy (BDS), ${ }^{11-14}$ of the changes on the segmental dynamics of NR when every vulcanizing additive has been added, and how the interactions between such additives and NR chains can help to elucidate the reactions involved in the sulfur vulcanization mechanism.

\section{EXPERIMENTAL}

NR used was kindly supplied by Malaysian Rubber (Berhad, Malaysia) under the trade name SMR CV60 (Mooney viscosity: $\left.\mathrm{ML}(1+4) 100^{\circ} \mathrm{C}=60\right)$. The compound formulation given on the basis of a total of 100 parts of rubber by weight (phr) is as follows: $\mathrm{S}$ ( $2.5 \mathrm{phr}$ ), ZnO (5 phr), SA (1 phr), and MBTS (mercapto benzothiazyl disulfide) (1 phr). All compounds were mixed in an open two-roll laboratory mill at room temperature, starting 
with the mastication of the rubber and then followed by the incorporation of the rest of ingredients. The prepared samples are referred to as $\mathrm{NR} / x / y / z$, where " $x$ ”, “ $y$ ”, and “ $z$ ", correspond to the additives present in the compound. It should be emphasized that in this study we are dealing with unvulcanized samples.

BDS measurements were performed on an ALPHA high resolution dielectric analyzer (Novocontrol Technologies GmbH, Hundsangen, Germany). The complex dielectric permittivity $\left(\varepsilon^{*}=\varepsilon^{\prime}-\mathrm{i} \varepsilon^{\prime \prime}\right)$ of the NR compounds was measured over a frequency window of $10^{-1}<\mathrm{F} / \mathrm{Hz}<10^{7}(\mathrm{~F}=\omega / 2 \pi$ is the frequency of the applied electric field $)$ in the temperature range from -100 to $100{ }^{\circ} \mathrm{C}$ in $5{ }^{\circ} \mathrm{C}$ steps. Samples of masticated neat NR and NR compounds as disk-shaped films were prepared by compression molding at room temperature. The disk samples were then mounted in the dielectric cell between two parallel gold-plated electrodes. Kapton ${ }^{\odot}$ spacers of $0.074 \mathrm{~mm}$ of thickness were used to prevent short circuits. ${ }^{15}$ Due to the use of spacers the measured values have to be considered as relative ones.

\section{RESULTS AND DISCUSSION}

Similarly to synthetic $1,4-$-cis-poly(isoprene) (PI), ${ }^{16-19}$ two distinct regions of dielectric dispersion are present in NR, ${ }^{11}$ a low temperature process (the segmental mode) related to the segmental motions of the polymer chain which has its origin in local motions of the perpendicular dipole moment; and a more intense process assigned to the normal mode which corresponds to motions of the entire chain caused by dipole components parallel to the chain backbone. In this research, we only analyze the segmental mode since the 
contribution of conductivity and interfacial polarization at low frequencies interfere in the analysis of the normal mode, as it can be seen in Figure 1. In this figure, a 3D representation of the frequency and temperature dependence of the dielectric loss $\varepsilon^{\prime \prime}$ for neat $\mathrm{NR}$ and $\mathrm{NR} / \mathrm{ZnO}$ compound is shown, for comparative purposes.
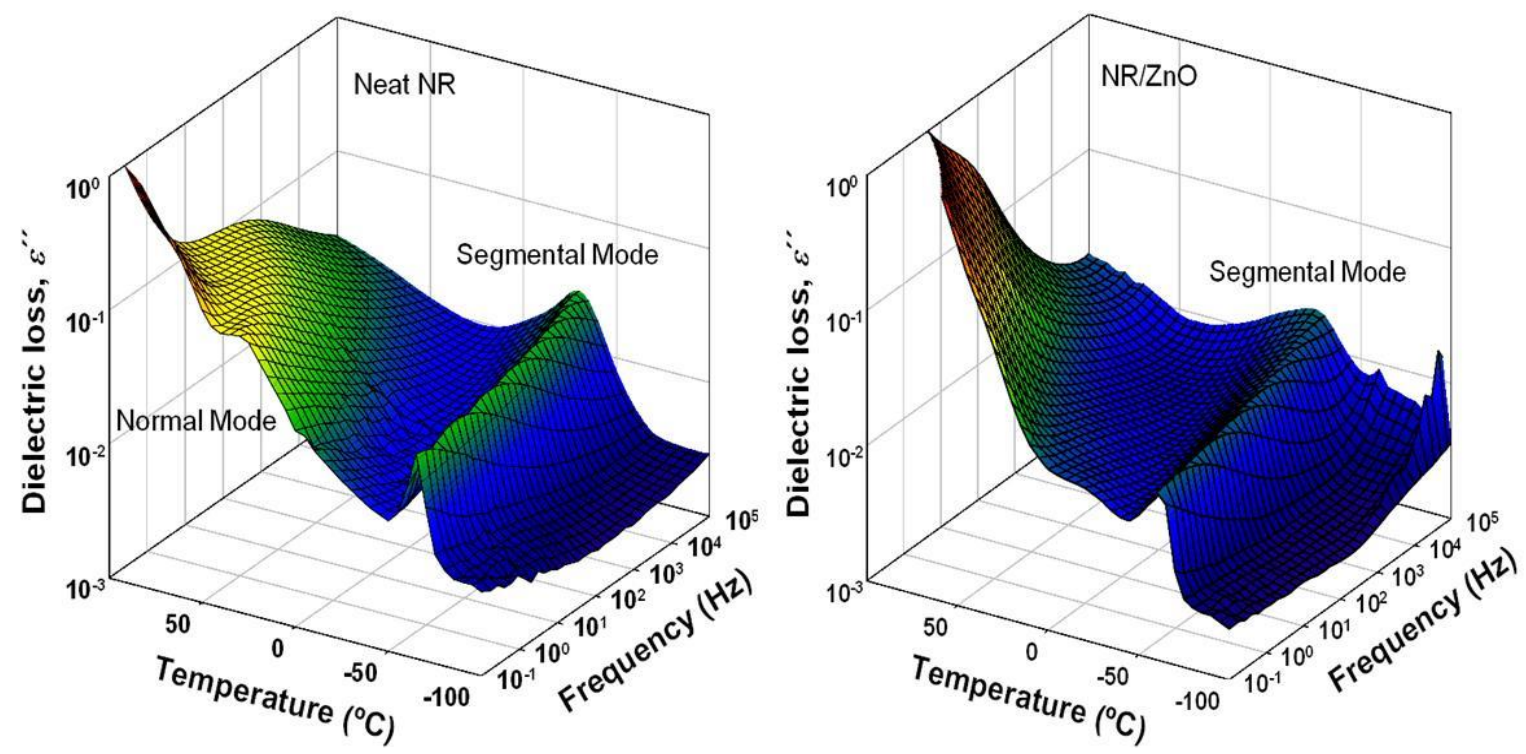

Figure 1. 3D representation of the frequency and temperature dependence of the dielectric $\operatorname{loss} \varepsilon^{\prime \prime}$ for neat $\mathrm{NR}$ and $\mathrm{NR} / \mathrm{ZnO}$ compound.

Figure 2 shows selected dielectric loss $\varepsilon^{\prime \prime}$ spectra at $\mathrm{T}=-50{ }^{\circ} \mathrm{C}$ for neat $\mathrm{NR}$ and $\mathrm{NR} /$ additives compounds measured in the available frequency range. From the spectra hereby presented, we can notice that the segmental mode process ( $\alpha$-relaxation) is well resolved in the frequency domain and manifests itself in all systems by a maximum in $\varepsilon^{\prime \prime}(\mathrm{F})$. We can also see that the presence of additives shifts the maximum of the loss peak to slightly lower frequencies, suggesting a restricted dynamics for the NR/additive compounds. For the sake of comparison and in order to avoid the effect of the non-absolute 
character of the measured $\varepsilon^{\prime \prime}$, the data have been normalized to the corresponding $\varepsilon^{\prime \prime}$ max of every curve. Here, we can notice a clear asymmetric broadening towards the low-frequency side of the dielectric loss curves.

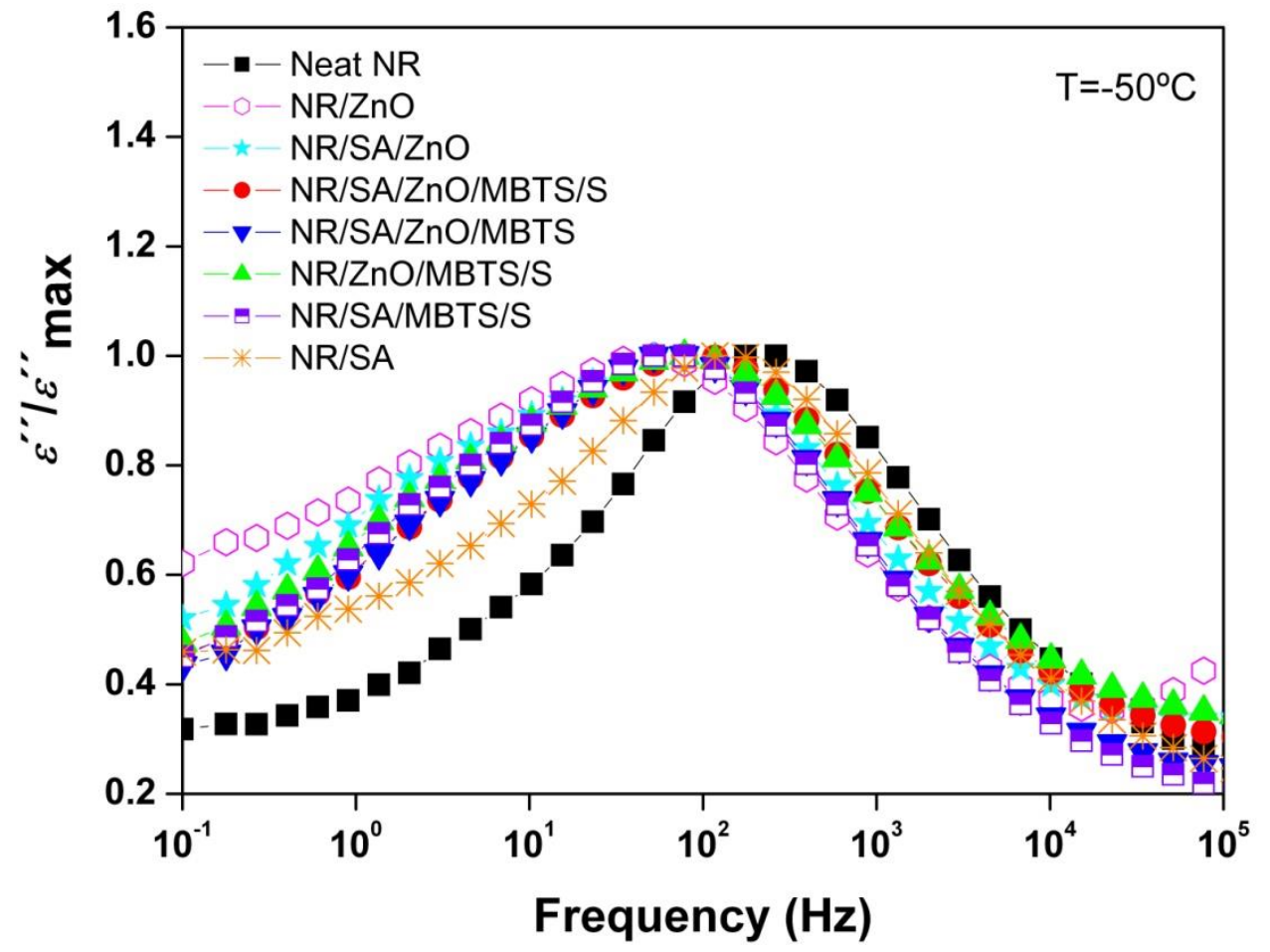

Figure 2. Normalized dielectric loss $\varepsilon^{\prime \prime}$ versus frequency of NR and its compounds in the region of the segmental mode.

Such a behavior suggests the existence of a second dynamic process slower but close to the segmental mode of NR appearing when additives are present. In one hand, it is worth to notice that the relaxation behavior of the NR/SA compound is the closest to the neat NR. 
On the other hand, the rest of the NR/additives compounds present a more pronounced broadening at the low frequency side of the dielectric loss curves.

In order to estimate the contribution of each additive in the relaxation process, every dielectric spectrum was analyzed and the experimental data of $\varepsilon^{\prime \prime}$ versus frequency was fitted in terms of the phenomenological Havriliak-Negami (HN) function ${ }^{20,21}$ described by:

$$
\varepsilon^{*}(\omega)=\varepsilon_{\infty}+\frac{\Delta \varepsilon}{\left[1+\left(i \omega \tau_{\mathrm{HN}}\right)^{b}\right]^{c}}
$$

Where $\Delta \varepsilon=\varepsilon_{S}-\varepsilon_{\infty}, \varepsilon_{\infty}$ and $\varepsilon_{s}$ are the unrelaxed and relaxed values of the dielectric constant and $b$ and $c$ are shape parameters $(0<b, c \leq 1)$ describing the symmetric and the asymmetric broadening of the equivalent relaxation time distribution function, respectively. $\tau_{\mathrm{HN}}$ is the Havriliak-Negami relaxation time, representing the most probable relaxation time of the relaxation time distribution function. ${ }^{22}$ The $\mathrm{HN}$ relaxation time is related to the frequency of maximum loss, $\mathrm{F}_{\max }=1 /\left(2 \pi \tau_{\max }\right)$, by the following equation: ${ }^{23}$

$$
\tau_{\max }=\frac{1}{2 \pi F_{\max }}=\tau_{\mathrm{HN}}\left[\sin \frac{b \pi}{2+2 c}\right]^{-1 / b}\left[\sin \frac{b c \pi}{2+2 c}\right]^{1 / b}
$$

Both characteristic relaxation times coincide when the relaxation spectrum is symmetric, $c=1$. 
The dielectric loss data were fitted by the superposition of two HN relaxation processes (named $\alpha^{\prime}$ and $\alpha$ in order of increasing frequency) corresponding to each one of the experimentally determined relaxations, plus a conductivity contribution (Figure 3).
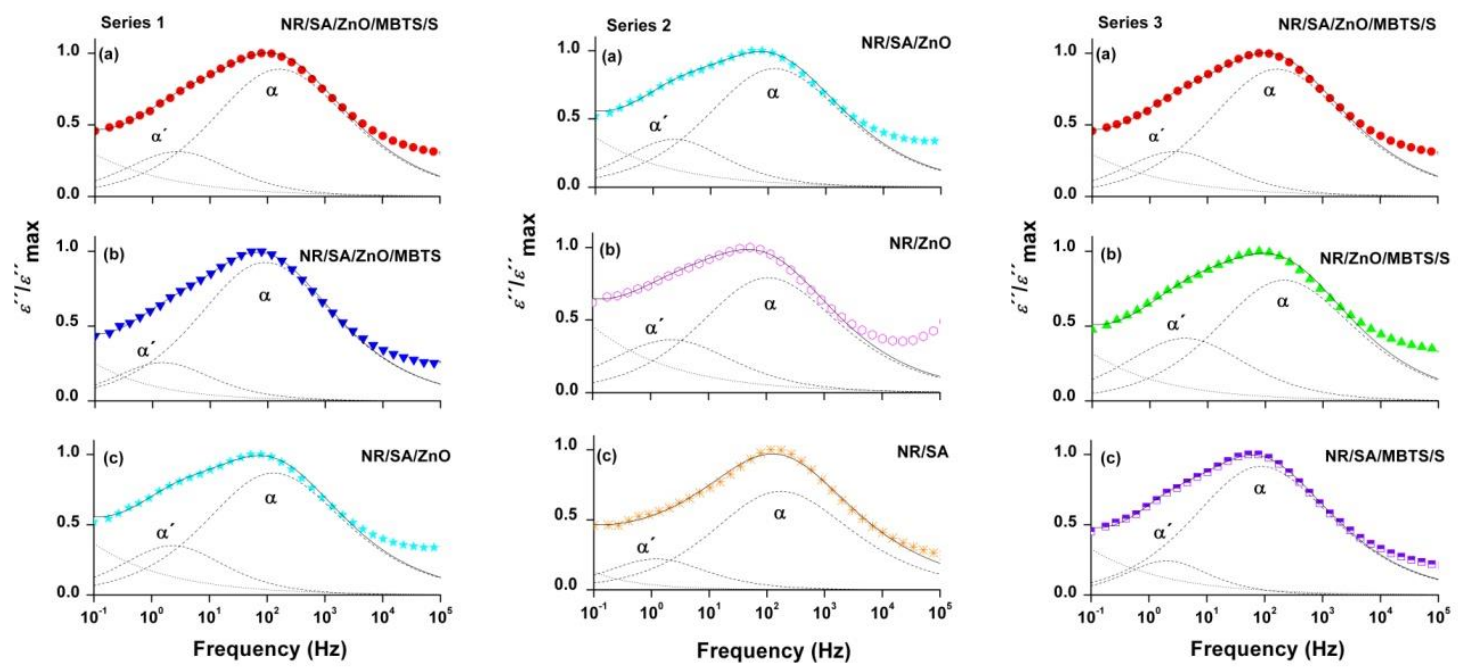

Figure 3. Deconvolution results for the normalized dielectric loss $\varepsilon^{\prime \prime}$ of NR compounds. Solid lines represent the HN fitting curve, dashed lines the individual processes and dotted lines the conductivity contribution.

The relaxation parameters $\left(\Delta \varepsilon, b, c\right.$ and $\left.\tau_{\mathrm{HN}}\right)$ representative of each fitting were calculated (Table 1). This fitting protocol was chosen in order to assure physical coherence. In a first approach, we assumed that the main relaxation process $(\alpha)$ was not significantly affected by the additives. Accordingly, we have considered a similar shape than that of the $\alpha$ relaxation of neat NR. Thus, the shape parameters $b$ and $c$ were kept constant and similar to those obtained for neat NR $(b=0.527 ; c=0.833)$. While for the $\alpha^{\prime}$-relaxation, the parameter $c$ 
was set to a value of 1 in order to force a symmetric shape and thus, a narrower distribution of relaxation times. Considering asymmetric shape for the $\alpha^{\prime}$ process rendered no reasonable fitting for the overall process. As expected, the strength relaxation intensity $(\Delta \varepsilon)$ is higher for the $\alpha$-relaxation than for the $\alpha^{\prime}$-relaxation for all compounds.

Table 1. Representative values of the fitting parameters of the HN function for all tested compounds at $\mathrm{T}=-50^{\circ} \mathrm{C}$.

\begin{tabular}{|c|c|c|c|c|}
\hline Compound & $\Delta \varepsilon_{\alpha}$ & $\Delta \varepsilon_{\alpha^{\prime}}$ & $\tau_{\mathrm{HN} \alpha}$ & $\tau_{\mathrm{HN \alpha}}{ }^{\prime}$ \\
\hline Neat NR & $1.431 \times 10^{-01}$ & --- & $1.045 \times 10^{-03}$ & --- \\
\hline $\mathrm{NR} / \mathrm{ZnO}$ & $6.699 \times 10^{-02}$ & $2.763 \times 10^{-02}$ & $2.062 \times 10^{-03}$ & $7.112 \times 10^{-02}$ \\
\hline $\mathrm{NR} / \mathrm{SA} / \mathrm{ZnO}$ & $6.514 \times 10^{-02}$ & $2.232 \times 10^{-02}$ & $1.599 \times 10^{-03}$ & $6.463 \times 10^{-02}$ \\
\hline $\mathrm{NR} / \mathrm{SA} / \mathrm{ZnO} / \mathrm{MBTS} / \mathrm{S}$ & $1.169 \times 10^{-01}$ & $3.211 \times 10^{-02}$ & $1.370 \times 10^{-03}$ & $5.799 \times 10^{-02}$ \\
\hline $\mathrm{NR} / \mathrm{SA} / \mathrm{ZnO} / \mathrm{MBTS}$ & $3.119 \times 10^{-02}$ & $8.782 \times 10^{-03}$ & $2.403 \times 10^{-03}$ & $1.115 \times 10^{-01}$ \\
\hline $\mathrm{NR} / \mathrm{ZnO} / \mathrm{MBTS} / \mathrm{S}$ & $6.355 \times 10^{-02}$ & $2.865 \times 10^{-02}$ & $1.048 \times 10^{-03}$ & $3.900 \times 10^{-02}$ \\
\hline NR/SA/MBTS/S & $7.785 \times 10^{-02}$ & $1.279 \times 10^{-02}$ & $2.556 \times 10^{-03}$ & $7.941 \times 10^{-02}$ \\
\hline $\mathrm{NR} / \mathrm{SA}$ & $4.975 \times 10^{-02}$ & $7.061 \times 10^{-03}$ & $1.328 \times 10^{-03}$ & $1.341 \times 10^{-01}$ \\
\hline
\end{tabular}


The dielectric loss spectra of the NR compounds have been grouped in Figure 3 as three different series, in order to analyze separately the effect of each of the additives on both relaxations ( $\alpha$ and $\alpha^{\prime}$ ). With Series 1 we intend to study the effect of sulfur (S) and accelerator (MBTS), while Series 2 and Series 3 results serve to elucidate the effect of the activator complex $(\mathrm{SA} / \mathrm{ZnO})$ with and without other additives present. Some of the figures shown in the Series are duplicated for the sake of clarity and better understanding of the systematic removal of the different vulcanizing additives.

From Figure 3-series 1 results, we can infer that sulfur (S) and accelerator (MBTS) are not likely to be responsible for the appearance of the slower process represented by $\alpha^{\prime}$. In this case, we can clearly see that after removing MBTS and S from the compound (series 1c), the shoulder at low frequencies is still present.

Next, we evaluate the effect of the activator complex (SA/ZnO) (Figure 3-series 2). Several authors $^{6,24-26}$ have studied the influence of the activator complex on the cross-linking reaction of NR. Recently, Ikeda et $\mathrm{al}^{5}$ have proposed that the combination of $\mathrm{ZnO}$ with the other reagents is crucial to control the quality of the vulcanization. Here we corroborate by BDS that $\mathrm{ZnO}$ is the element with the strongest effect on the segmental dynamics of NR. In this sense, the low frequency shoulder characterized by $\alpha^{\prime}$ appears when the complex, $\mathrm{SA} / \mathrm{ZnO}$, is present (Figure 3-series 2a). However, the elimination of SA from the formulation leaves $\alpha^{\prime}$ almost unaffected (Figure 3-series 2b). The origin of this slow relaxation in NR has been discussed in the literature. In a previous study, Ortiz-Serna et al. ${ }^{27}$ attributed this slower relaxation to the dynamics of SA linked to the polymer chains. Our measurements indicate that this slower relaxation is mainly attributed to the presence of $\mathrm{ZnO}$. 
The influence of $\mathrm{ZnO}$ on the dielectric behavior of polymer matrices has been discussed. Psarras et al. ${ }^{28,29}$ found an additional relaxation mode related to the presence of $\mathrm{ZnO}$ in epoxy resin- $\mathrm{ZnO}$ composites, in the same frequency and temperature range as the $\alpha$ relaxation, leading to a mutual superposition. They attributed this behavior to polarization effects taking place in parts of the $\mathrm{ZnO}$, which are relaxing under the influence of the $\mathrm{AC}$ electric field. Also, Smaoui et al. $^{30}$ found that the presence of $\mathrm{ZnO}$ in epoxy nanocomposites increases the interactions at the interface, so decreasing the orientation capabilities of dipoles under the effect of an AC electric field.

$\mathrm{ZnO}$ is known as the best activator for sulfur vulcanization. There is nowadays, however, an increasing concern regarding the potential environmental and health effects of releasing zinc compounds into the environment from rubber products or rubber production. ${ }^{31}$ Thus, it is desirable to keep the $\mathrm{ZnO}$ content in rubber compounds as low as possible, not only for environmental but also for economical reasons. Nonetheless, the exact role of $\mathrm{ZnO}$ on the network structure has not been well understood. Several theories have been postulated, and the complexity of the vulcanization process has given rise to many uncertainties and contradictions in the literature about the influence of $\mathrm{ZnO}$ during the different stages of the process and its exact mechanisms. ${ }^{2,3,32}$ One of the proposed mechanisms ${ }^{2,7,33}$ suggest that $\mathrm{ZnO}$ plays the role of a catalyst and promotes the initial response by activating and bringing together reactants. It is assumed that $\mathrm{ZnO}$ is distributed in the form of crystal particles in the rubber compounds. Molecules of accelerators, sulfur and fatty acid are adsorbed on the surface of the $\mathrm{ZnO}$, forming intermediate complexes (benzothiazole-zinc complex). The reactant of the benzothiazole-zinc complex does not have appreciable solubility in rubber, but the solubility and reactivity can be enhanced by the presence of SA, forming zinc 
stearate. ${ }^{5,25}$ Some authors ${ }^{4}$ suggest that the ionic nature of zinc stearate is responsible for the appearance of a second relaxation process in vulcanized NR, which may be attributed to rubber chains of lower mobility tightly bounded to zinc stearate surface. It is known that in the case of ion-containing polymers, ion pairs segregate to form ion aggregates, which restrict the mobility of adjacent segments of the polymer chains and form a separate rigid phase. $^{34,35}$ These immobile segments of the rubber chains undergo relaxation at lower frequencies. In other words, this means that, there are some restrictions on the molecular dynamics of this process limiting the motion of NR chains in their surrounding environment. Thus, these BDS results are strong evidence of the activator effect that $\mathrm{ZnO}$ has on the vulcanization process of NR, since the $\alpha^{\prime}$-relaxation could be attributed to the ionic clusters of $\mathrm{ZnO}$ that could form initial physical entanglements within the rubber chains, acting as crosslink precursors, as schematically represented in Figure 4. Results from Figure 3-Series 3 confirm that the absence of $\mathrm{ZnO}$ in the formulation gives an $\alpha^{\prime}$ relaxation of very low intensity. 


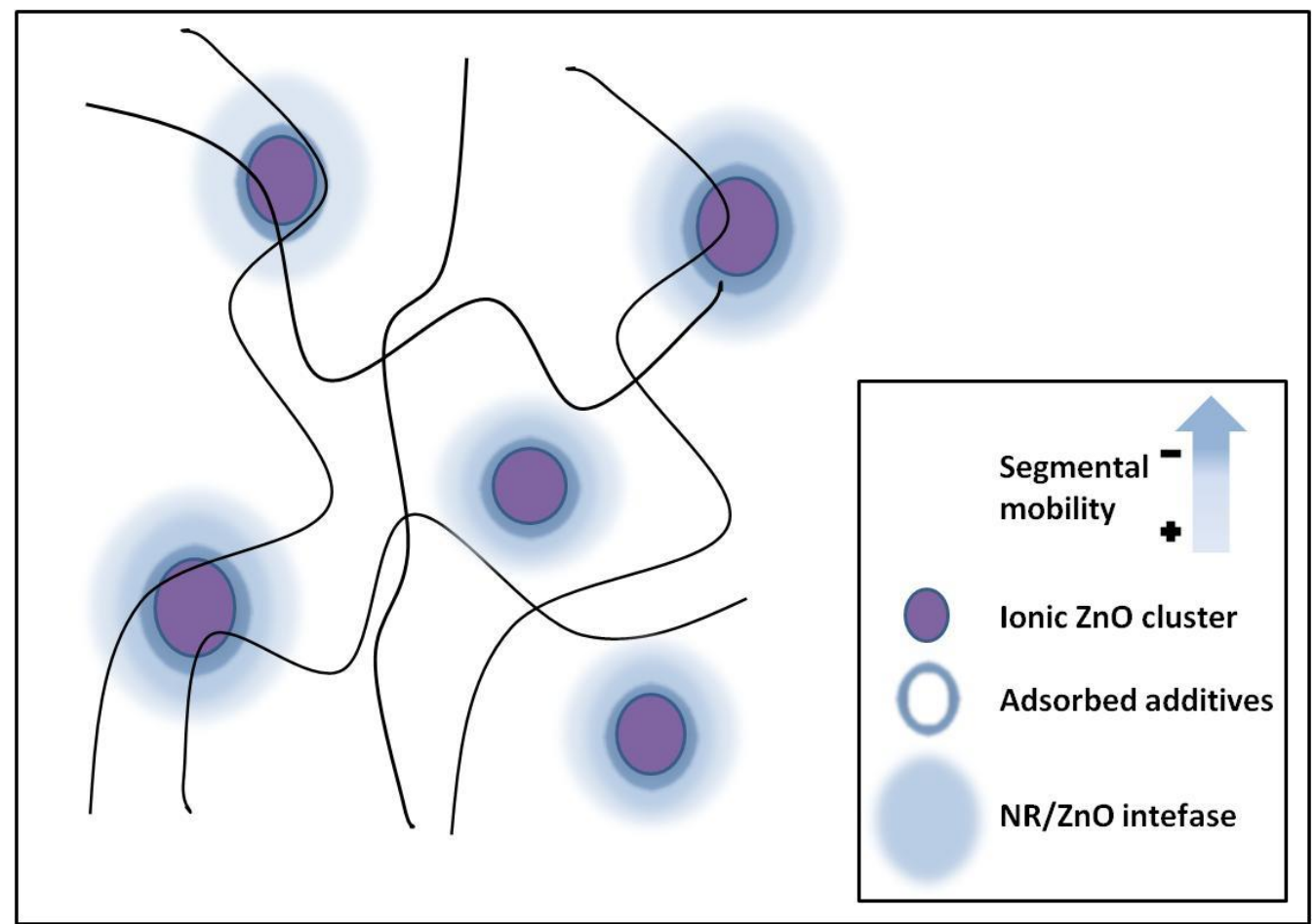

Figure 4. Proposed schematic representation of morphological structure of NR/additives compound.

In Figure 5 we present an intensity relation chart between the dielectric strength values of both relaxation processes applying different fitting protocols in order to establish the merits of our previous fitting assumptions. It should be noted that the error bars presented in the graph do not correspond to the experimental error but arise from the spread of the fitting parameters applied. It is clear that the origin of the slower process present in the NR compounds is directly associated with the presence of the different additives. However, two interesting results can be extracted from the analysis of this chart. First of all, the NR/SA compound has the lower intensity relation value, so we can presume that the contribution of the SA to the $\alpha^{\prime}$-relaxation is insignificant. This fact is in agreement with the results shown 
in Figure 2, where, as previously mentioned, there is certain similarity between the normalized loss curves of neat NR and NR/SA compound.

The second interesting result extracted from Figure 5 is the higher intensity relation value obtained for all compounds where $\mathrm{ZnO}$ is present. Thus, it clearly implies that the origin of the slower process present in the NR compounds is directly associated with the presence of $\mathrm{ZnO}$.

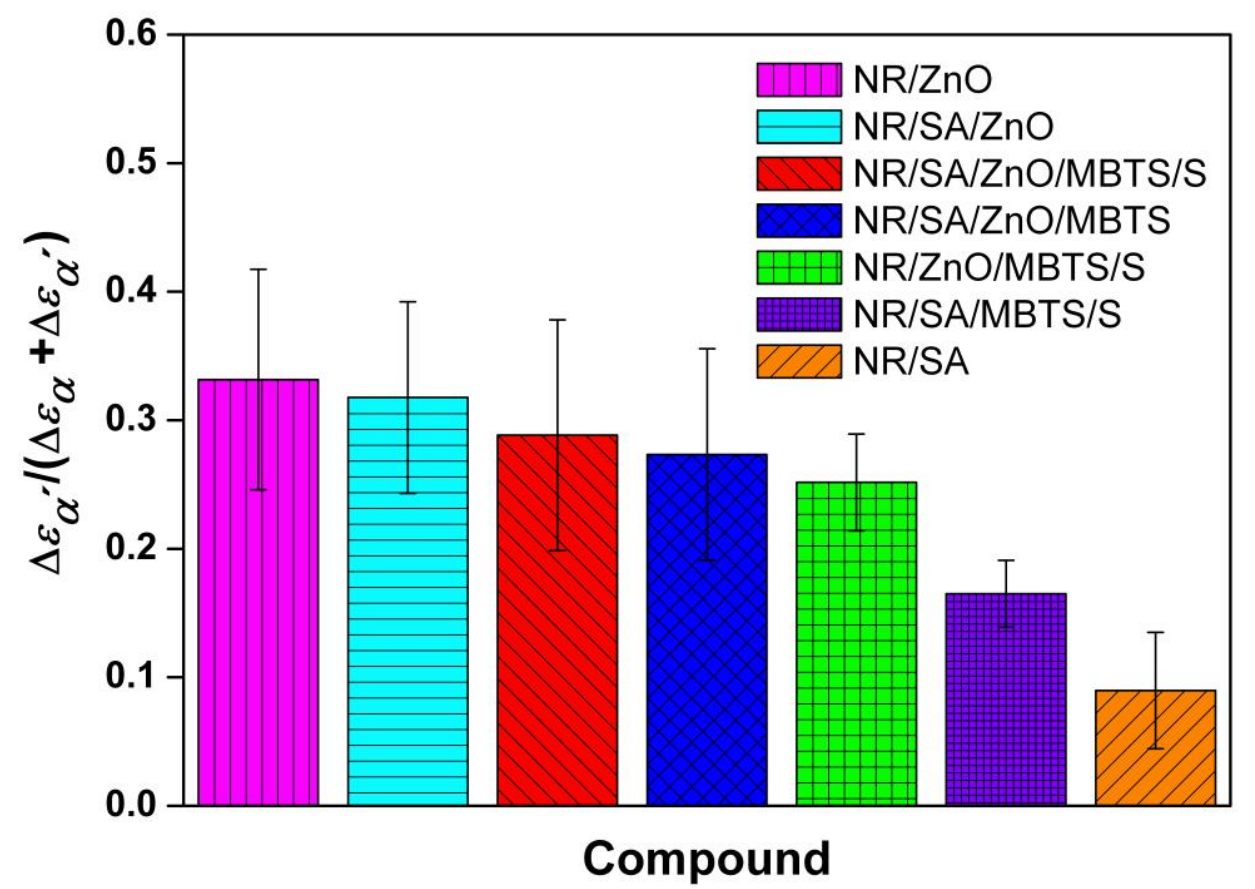

Figure 5. Dielectric strength intensity relation of the $\alpha$ - and $\alpha^{\prime}$ - relaxations of NR compounds. Bars correspond to average values representative of different possible $\mathrm{HN}$ fittings. 


\section{CONCLUSIONS}

In summary, we have made an experimental study of the influence of the addition of vulcanizing components on the molecular dynamics of NR. Our results reveal a slowdown of the segmental dynamics of NR restricting the motion of rubber chains tightly bounded to additive surfaces. In general, when $\mathrm{ZnO}$ is present a visible second dynamic process is detected. Systematic removal of the different vulcanizing additives leads us to ascribe this slow process to strong interfacial interactions formed at the interfaces between the $\mathrm{ZnO}$ ionic clusters and the NR polymer segments, forming initial physical entanglements between both components, which may act as crosslinking precursors. We believe that these results can help to understand basic issues related to the network structure and molecular mobility of NR, essentials for the optimization of the vulcanization process, the role of additives and their interactions with the NR matrix. Moreover, the better understanding of the role of $\mathrm{ZnO}$ in the vulcanization reaction is a key issue for the ongoing efforts to reduce zinc content of vulcanization mixtures without reducing the overall quality of the products.

\section{ACKNOWLEDGEMENTS}

The authors gratefully acknowledge the financial support of the MCINN (grants MAT2009-07789 and MAT2010-18749). M. Hernández thanks the Venezuelan Ministry of Science and Technology for the concession of a "Mision Ciencia" grant. 


\section{REFERENCES}

1. Brydson, J. A., Rubbery Materials and Their Compounds. Elsevier Science Publishers Ltd: London, 1988; p 469.

2. Heideman, G.; Datta, R. N.; Noordermeer, J. W. M.; Van Baarle, B. Rubber Chem. Technol. 2004, 77, (3), 512-541.

3. Steudel, R.; Steudel, Y. Chem. Eur. J. 2006, 12, (33), 8589-8602.

4. Ward, A. A.; El-Sabbagh, S.; El-Aal, N. S. A. KGK-Kautsch. Gummi Kunstst. 2008, 61, (9), 429-437.

5. Ikeda, Y.; Higashitani, N.; Hijikata, K.; Kokubo, Y.; Morita, Y.; Shibayama, M.; Osaka, N.; Suzuki, T.; Endo, H.; Kohjiya, S. Macromolecules 2009, 42, (7), 2741-2748.

6. Poh, B. T.; Tang, W. L. J. Appl. Polym. Sci. 1995, 55, (3), 537-542.

7. Kim, I. J.; Kim, W. S.; Lee, D. H.; Kim, W.; Bae, J. W. J. Appl. Polym. Sci. 2010, 117, (3), 1535-1543.

8. Cavdar, S.; Ozdemir, T.; Usanmaz, A. Plast. Rubber Compos. 2010, 39, (6), 277282.

9. Kohjiya, S.; Tosaka, M.; Masahiro, F.; Ikeda, Y.; Toki, S.; Hsiao, B. S. Polymer 2007, 48, 3801-3808.

10. Hofmann, W., Vulcanization and Vulcanizing Agents. Maclaren: 1967; p 371.

11. Hernandez, M.; Carretero-Gonzalez, J.; Verdejo, R.; Ezquerra, T. A.; LopezManchado, M. A. Macromolecules 2010, 43, (2), 643-651.

12. Carretero-Gonzalez, J.; Ezquerra, T. A.; Amnuaypornsri, S.; Toki, S.; Verdejo, R.; Sanz, A.; Sakdapipanich, J.; Hsiao, B. S.; Lopez-Manchado, M. A. Soft Matter 2010, 6, (15), 3636-3642. 
13. Boese, D.; Kremer, F. Macromolecules 1990, 23, (3), 829-835.

14. Hernandez, M.; Lopez-Manchado, M.; Sanz, A.; Nogales, A.; Ezquerra, T. A. Macromolecules 2011.

15. Sics, I.; Ezquerra, T. A.; Nogales, A.; Balta-Calleja, F. J.; Kalnins, M.; Tupureina, V. Biomacromolecules 2001, 2, (2), 581-587.

16. Mijovic, J.; Lee, H. K.; Kenny, J.; Mays, J. Macromolecules 2006, 39, (6), 21722182.

17. Boese, D.; Kremer, F. Macromolecules 1990, 23, (3), 829-835.

18. Boese, D.; Kremer, F.; Fetters, L. J. Macromolecules 1990, 23, (6), 1826-1830.

19. Page, K. A.; Adachi, K. Polymer 2006, 47, (18), 6406-6413.

20. Havriliak.S; Negami, S. Polymer 1967, 8, (4), 161-210.

21. Kremer, F.; Schönhals, A., Broadband Dielectric Spectroscopy. Springer: New York, 2003; p 721.

22. Böttcher, C. J. F.; Bordewijk, P., Theory of Electric Polarization. Elsevier: 1978; Vol. II.

23. Richert, R.; Angell, C. A. J. Chem. Phys. 1998, 108, (21), 9016-9026.

24. Allan, J. R.; Geddes, W. C.; Hindle, C. S.; Lowe, A. J. Plast. Rubber Compos. Process. Appl. 1991, 16, (2), 91-94.

25. Zhao, F.; Zhang, P.; Zhao, S. G.; Yu, J.; Kuhn, W. KGK, Kautsch. Gummi Kunstst. 2008, 61, (5), 224-229.

26. Helaly, F. M.; El Sabbagh, S. H.; El Kinawy, O. S.; El Sawy, S. M. Mater. Des. 2011, 32, (5), 2835-2843.

27. Ortiz-Serna, P.; Diaz-Calleja, R.; Sanchis, M. J.; Floudas, G.; Nunes, R. C.; Martins, A. F.; Visconte, L. L. Macromolecules 2010, 43, (11), 5094-5102. 
28. Soulintzis, A.; Kontos, G.; Karahaliou, P.; Psarras, G. C.; Georga, S. N.; Krontiras, C. A. J. Polym. Sci. Pt. B-Polym. Phys. 2009, 47, (4), 445-454.

29. Ioannou, G.; Patsidis, A.; Psarras, G. C. Compos/Part A: Appl. Sci. Manuf. 2011, 42, (1), 104-110.

30. Smaoui, H.; Mir, L. E. L.; Guermazi, H.; Agnel, S.; Toureille, A. J. Alloys Compd. 2009, 477, (1-2), 316-321.

31. Chapman, A. V. Safe Rubber Chemicals: Reduction of Zinc Levels in Rubber Compounds; TARRC/MRPRA: 1997.

32. Heideman, G.; Datta, R. N.; Noordermeer, J. W. M.; van Baarle, B. J. Appl. Polym. Sci. 2005, 95, (6), 1388-1404.

33. Nieuwenhuizen, P. J.; Timal, S.; Van Veen, J. M.; Haasnoot, J. G.; Reedijk, J. Rubber Chem. Technol. 1998, 71, (4), 750-765.

34. Antony, P.; Bandyopadhyay, S.; De, S. K. Polym. Eng. Sci. 1999, 39, (5), 963-974.

35. Hird, B.; Eisenberg, A. Macromolecules 1992, 25, (24), 6466-6474. 
"for Table of Contents use only"

\section{Role of Vulcanizing Additives on the Segmental Dynamics of Natural Rubber}

Marianella Hernández, Tiberio A. Ezquerra, Raquel Verdejo, Miguel A. López-Manchado

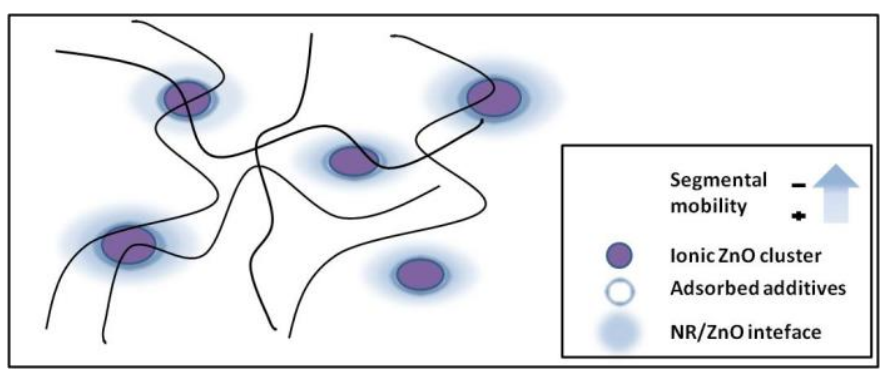

